\title{
Antimicrobial Activity and Characterization of Pomegranate Peel-Based Carbon Dots
}

\author{
Waseem Akhtar Qureshi $\mathbb{D}^{1},{ }^{1}$ B. Vivekanandan $(\mathbb{D})^{2}$ J. Altrin Jayaprasath $\mathbb{D}^{\circ},{ }^{3}$ Daoud Ali $(\mathbb{D})^{4}$ \\ Saud Alarifi ${ }_{(1)}{ }^{4}$ and Kalim Deshmukh ${ }^{5}$ \\ ${ }^{1}$ Cholistan Institute of Desert Studies, Baghdad-ul-Jadeed Campus, The Islamia University of Bahawalpur, Pakistan \\ ${ }^{2}$ Department of Chemical Engineering, Hindustan Institute of Technology and Science, 603103, Chennai, Tamil Nadu, India \\ ${ }^{3}$ Department of Botany, Voorhees College, 632001, Vellore, Tamil Nadu, India \\ ${ }^{4}$ Department of Zoology, College of Science, King Saud University, PO Box 2455, Riyadh 11451, Saudi Arabia \\ ${ }^{5}$ New Technologies - Research Center, University of West Bohemia, Plzeň 30100, Czech Republic \\ Correspondence should be addressed to Daoud Ali; daoudali08@yahoo.com
}

Received 9 September 2021; Accepted 4 October 2021; Published 11 November 2021

Academic Editor: Lakshmipathy R

Copyright (C) 2021 Waseem Akhtar Qureshi et al. This is an open access article distributed under the Creative Commons Attribution License, which permits unrestricted use, distribution, and reproduction in any medium, provided the original work is properly cited.

\begin{abstract}
This investigation reports the use of agrowaste pomegranate peels as an economical source for the production of fluorescent carbon dots (C-dots) and their potential application as an antimicrobial agent. The carbon dots were prepared through low-temperature carbonization at $200^{\circ} \mathrm{C}$ for $120 \mathrm{~min}$. The obtained $\mathrm{C}$-dots were found to be small in size and exhibited blue luminescence at $350 \mathrm{~nm}$. Further, the synthesized C-dots were characterized with the help of analytical instruments such as DLS, UV-visible, FT-IR, TEM, and fluorescence spectrophotometer. Antimicrobial activity of the C-dot PP was estimated by the agar diffusion method and MIC. S. aureus and K. pneumoniae are showing susceptibility towards C-dot PP when compared to the standard and showing a moderate activity against P. aeruginosa and resistance towards E. coli. The obtained C dot PPs were found to be around 5-9 nm in size confirmed from DLS analysis and supported by TEM. The synthesized C-dots were investigated to understand their microbial efficiency against pathogens and found to have antimicrobial efficiency. These results suggest that pomegranate peels are a potential source of carbon dots with antimicrobial efficiency.
\end{abstract}

\section{Introduction}

Nanoparticles (NPs) have become indispensable in biological research due to long-term fluorescence imaging and identification of their properties. Photobleaching resistance, improved signal brightness, larger absorption coefficients, light emission, and simultaneous stimulation of multiple fluorescence colours are just a few of the unique properties of QDs that make them indispensable [1]. Guan et al. created bright $\mathrm{C}$-dots by using folic acid molecules as both nitrogen and carbon donors [2].

Carbon dots are promising oxygenous carbon nanoparticles that exhibit photoluminescence properties. These dots can replace quantum dots in semiconductor devices and some future nanodevices because of their high water solubility, tiny size $(<10 \mathrm{~nm})$, intense brightness, high photostability, lesser cytotoxicity, and biocompatibility [3, 4]. The applications of carbon dots are diverse and found to be prolific in nature starting from pollutant detection to battery electrodes and markers [5-8]. However, the production of carbon dots involves tedious processes that are detrimental to the environment and ecosystems. The previously reported methods have many drawbacks such as the use of chemicals, high temperature, and low product yield. Researchers are always in search of new and better ways of producing C-dots [9]. In a growing number of publications, plant by-products such as exotic fruit by-products as a source of food additives, fruit by-product extracts as antioxidants 


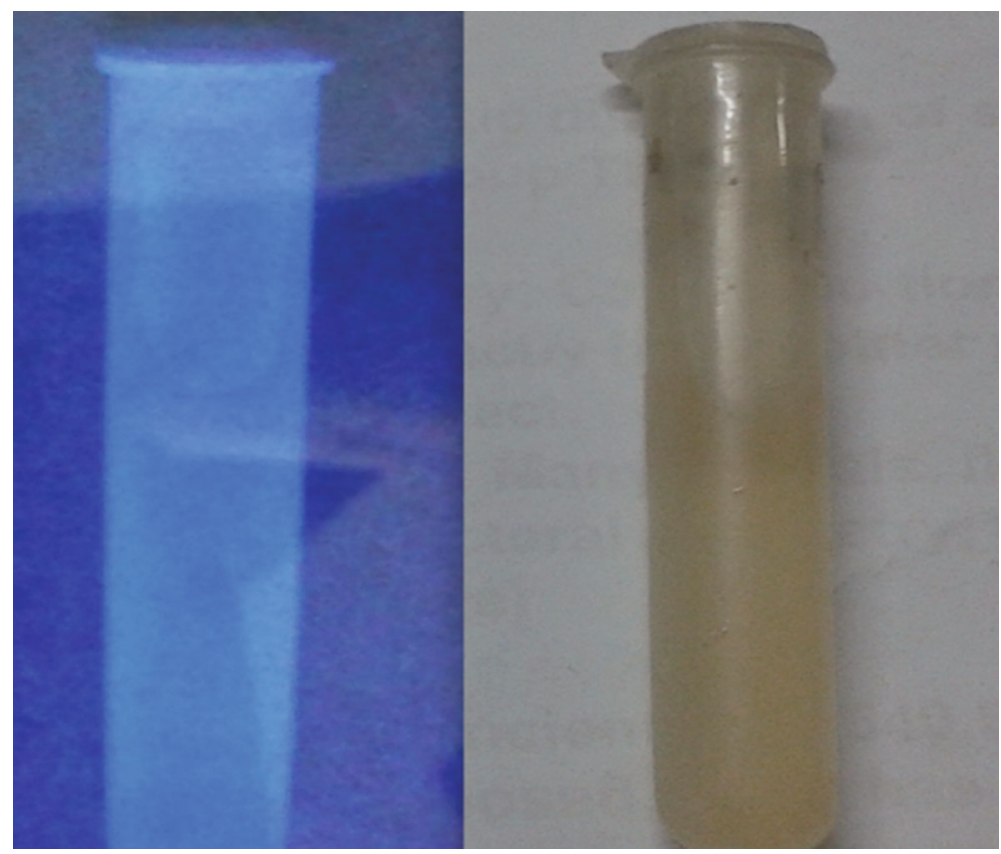

Figure 1: Fluorescence exhibited at $350 \mathrm{~nm}$ by carbon dots.

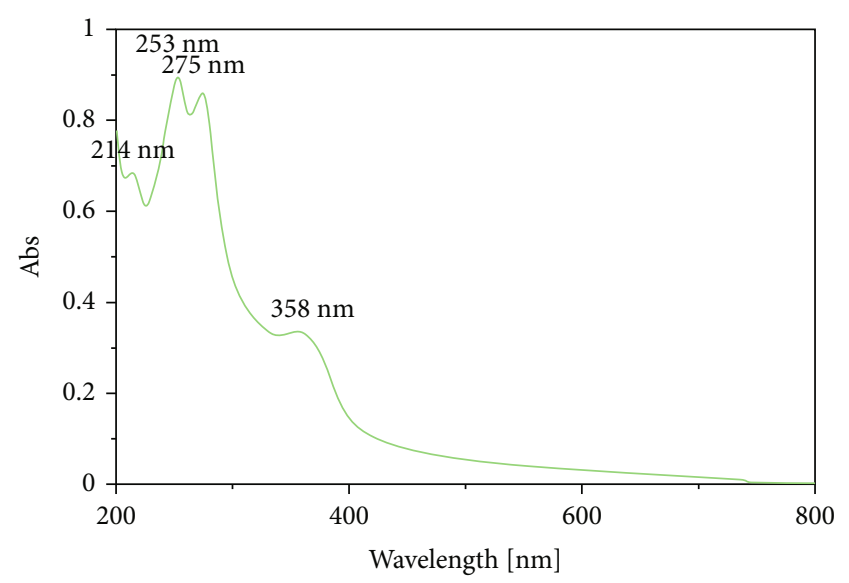

Figure 2: UV-visible absorption spectrum of C-dots prepared from pomegranate peels.

and antibacterial [10]. Plant wastes are appealing starting materials for CQDs because of their biocompatibility, low cost, and low toxicity [11].

Agrowastes are one of the potential sources for the production of carbon dots, and many fruit wastes are explored for this purpose [12-15]. Since the fruit wastes are easily available, low-cost, and eco-friendly, they have gained significance in the synthesis of carbon dots [14]. In continuation of the above, here, we report a new agrowaste fruit peelbased material (pomegranate peel) for the synthesis of carbon dots. Pomegranate is a delicious fruit with hard outer cover that is carbonaceous in nature. The peels are usually discarded, and this carbonaceous material can be a great precursor for the carbon dots.
The aim of this investigation is to produce C-dots from pomegranate peels through a low-temperature carbonization and filtration process. This method is extremely environment-friendly, and it does not involve any toxic or harmful chemical. The materials used for the synthesis are cheap and easily available and biowaste. The obtained C-dots are water soluble and highly fluorescent and have been investigated for antimicrobial activity.

\section{Experiment}

2.1. Synthesis of Fluorescent C-Dots. The fresh pomegranate peels sourced from local fruit vendors were cut into tiny pieces and dried in the sunlight to remove the moisture contents. The sun dried samples were then carbonized at $200^{\circ} \mathrm{C}$ for 2 hours. One gram of carbonized pomegranate peels was weighed and dispersed in $100 \mathrm{ml}$ ultrapure water and sonicated for 15 minutes. The sonicated solution was later filtered with the help of a filter paper to obtain the supernatant solution containing carbon dots. Then, the superannuated solution was centrifuged, and the resultant supernate containing luminescent C-dots was analysed by various analytical methods.

\subsection{Characterization}

2.2.1. UV-Vis Spectral Analysis. The experimental absorption spectrum of compounds in the $200-700 \mathrm{~nm}$ regions in unpolarised light was obtained by using a JASCO V-670 UV-visible spectrophotometer. The estimated and observed absorption spectra demonstrate the accuracy of the derived model calculations. 


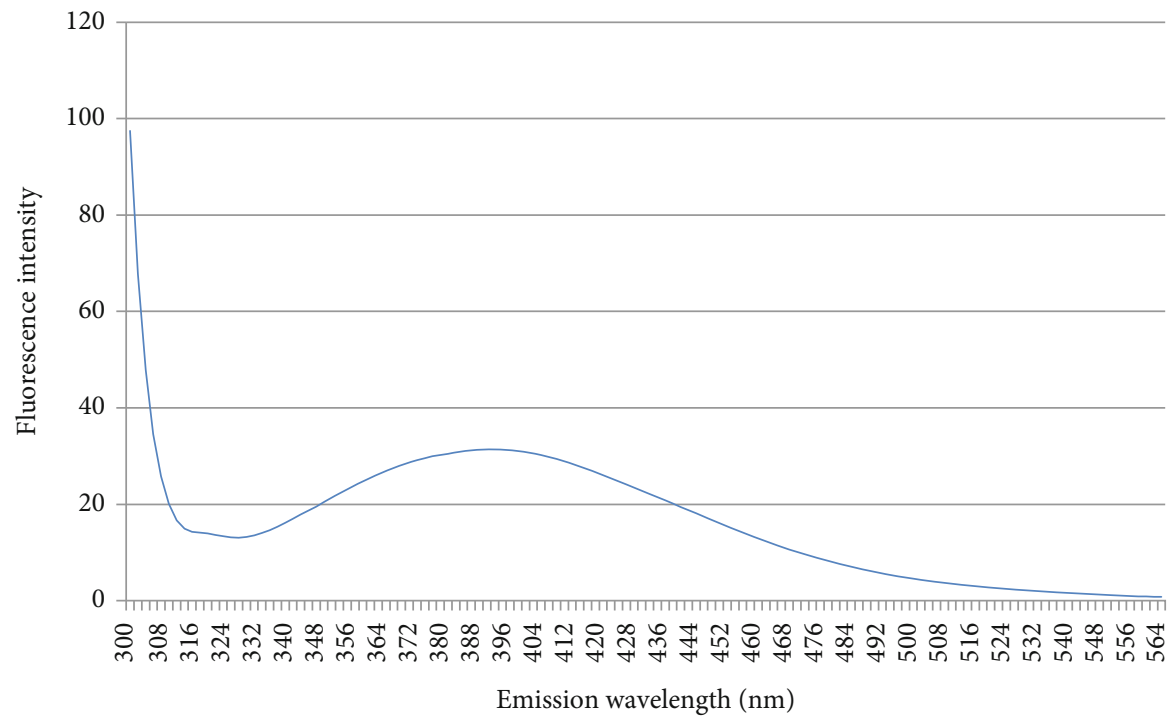

Figure 3: Emission spectra of C-dots prepared from pomegranate peels.

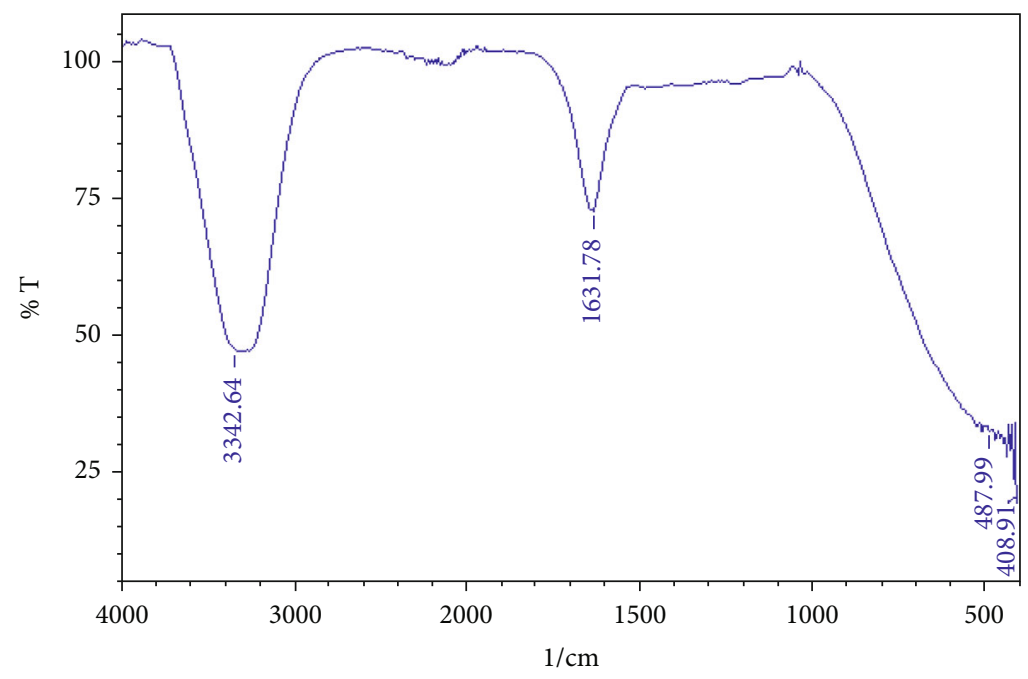

FIGURE 4: FT-IR spectrum of C-dots prepared from pomegranate peels.

2.2.2. Fluorescence Spectroscopy. A Hitachi F-7000 fluorescence spectrometer was equipped to obtain the fluorescence spectra as it has a high sensitivity measurement due to a design that prohibits fluorescence detecting along the flow channel.

2.2.3. FT-IR Analysis. FT-IR spectroscopy has proven to be a worldwide, sensitive, and highly repeatable physicochemical analytical approach that uses infrared absorption to identify the functional groups. FT-IR spectrum was obtained with an IR Affinity-1 (Shimadzu) between 400 and $4000 \mathrm{~cm}^{-1}$. The samples were recorded on a $\mathrm{KBr}$ disc prepared freshly.

2.2.4. Particle Size and Zeta Potential. Using a Malvern Zetasizer Nano ZS (Malvern Instruments, Ltd., Malvern, Wor- cestershire, UK) equipped with a $\mathrm{Ne}-\mathrm{He}$ laser, particle size was determined by dynamic light scattering $(633 \mathrm{~nm})$. According to the equipment specifications, measurements were taken at a fixed angle of $173^{\circ}$ from $0.6 \mathrm{~nm}$ to $6 \mathrm{~m}$. PC samples $\left(5 \mathrm{mg} \mathrm{ml}^{-1}\right)$ were diluted to $1: 100$ in Milli-Q water and placed in disposable polaroid containers.

2.2.5. TEM (Transmission Electron Microscope). TEM was recorded with a JEOL JEM 2100F instrument operated at $200 \mathrm{kV}$. The diluted solution was placed over carboncoated copper grids and dried outside. A total of ten sites on the TEM grid were examined.

2.2.6. Antimicrobial Activity. Four isolates such as Staphylococcus aureus, Klebsiella pneumoniae, Pseudomonas 


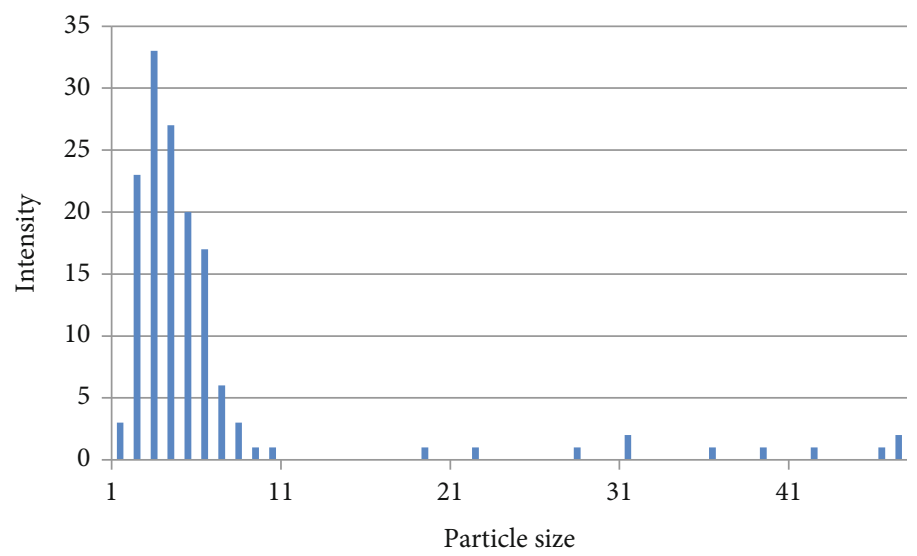

FIGURE 5: DLS pattern of C-dots prepared from pomegranate peels.

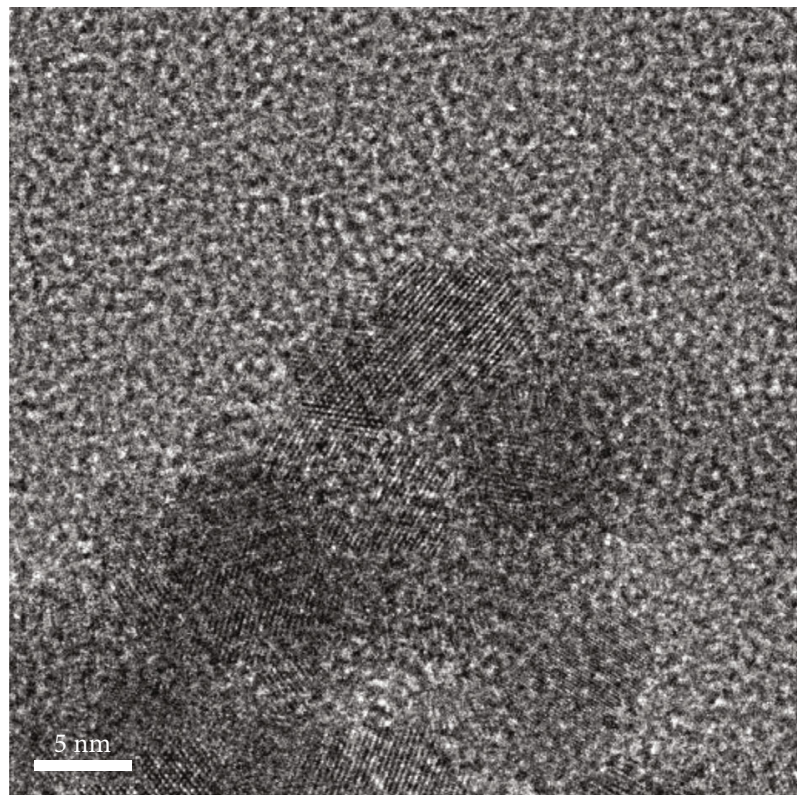

FIGURE 6: TEM image of C-dots prepared from pomegranate peels.

aeruginosa, and Escherichia coli were collected from the Department of Microbiology and Pathology, VIT, Tamil $\mathrm{Nadu}$, India. The collected isolates were stocked and stored at $-20^{\circ} \mathrm{C}$ in glycerol.

2.2.7. Agar Diffusion Method. Agar well diffusion on Mueller Hinton Agar, a popular method for antimicrobial screening, was adopted for pathogenic strains [15]. The incubation of isolates was carried out at $37^{\circ} \mathrm{C}$ for 24 hours in a nutrient broth with turbidity 0.5 adjusted with a spectrophotometer. The MHA plates were selected for the investigations, and wells were made with a sterilized gel borer and bacterial isolates of Actinobacteria were inoculated with the help of cotton. The solvent and carbon dot solution were tested against a test pathogen by adding $100 \mu \mathrm{l}$ of test solutions into each well and ampicillin $(30 \mu \mathrm{g} / \mathrm{ml})$ as the standard. After incubation, the zone of inhibition developed around the wells in all the plates was examined [15].
2.2.8. Minimum Inhibitory Concentration (MIC). A tube dilution method was adopted to understand the MIC of the potent Actinobacteria. Carbon dot solution was diluted in $10 \%$ DMSO to obtain various concentrations ranging from 5 to $100 \mu \mathrm{g} / \mathrm{ml}$ of test solutions. Inoculation of $0.05 \mathrm{ml}$ of bacterial culture was done by the addition of $5 \mathrm{ml}$ of sterile Mueller Hinton Broth. To all the test tubes, $1 \mathrm{ml}$ of C-dot PP solution was added and incubated for $24 \mathrm{~h}$ in order to observe any development of turbidity. The minimal concentration that could inhibit complete growth is considered MIC [16].

\section{Results and Discussion}

Carbon quantum dots (CQDs) have received a lot of attention in recent years because of their outstanding physical and optical properties. There are numerous types of carbon sources used in various fields, but CQDs used in the food industry must meet tighter safety standards. As a result, using 24 natural resources to prepare CQDs without involving humans is the best alternative [17]. Quantum dots, which range in size from 1 to 10 nanometers, are luminous semiconductor nanocrystals with complicated surface chemistry and unique optical properties (QDs). C-dots have optical features that allow them to reflect hits from particles of varying sizes in the sample [1].

The successfully synthesized C-dots from pomegranate peels were characterized for it properties with various analytical techniques. Preliminary investigations were made to confirm the formation of carbon dots with the help of the UV chamber. The samples were placed in the UV chamber, and UV light $(354 \mathrm{~nm})$ was passed on the tube containing carbon dot solution. A blue fluorescence was noticed at $354 \mathrm{~nm}$ which confirmed the presence of carbon dots. In the absence of UV light, ordinary solution was observed to have no fluorescence (Figure 1). Further, UV-vis analysis was carried out to observe the absorption spectra. An absorption peak at 214, 253, 275, and $358 \mathrm{~nm}$ was observed for the carbon dot solution; the peaks at 214 and $253 \mathrm{~nm}$ confirm the presence of carbon dots and light absorption due to sigma-sigma transitions (Figure 2). The absorption peak at $358 \mathrm{~nm}$ observed could be due to the presence of 


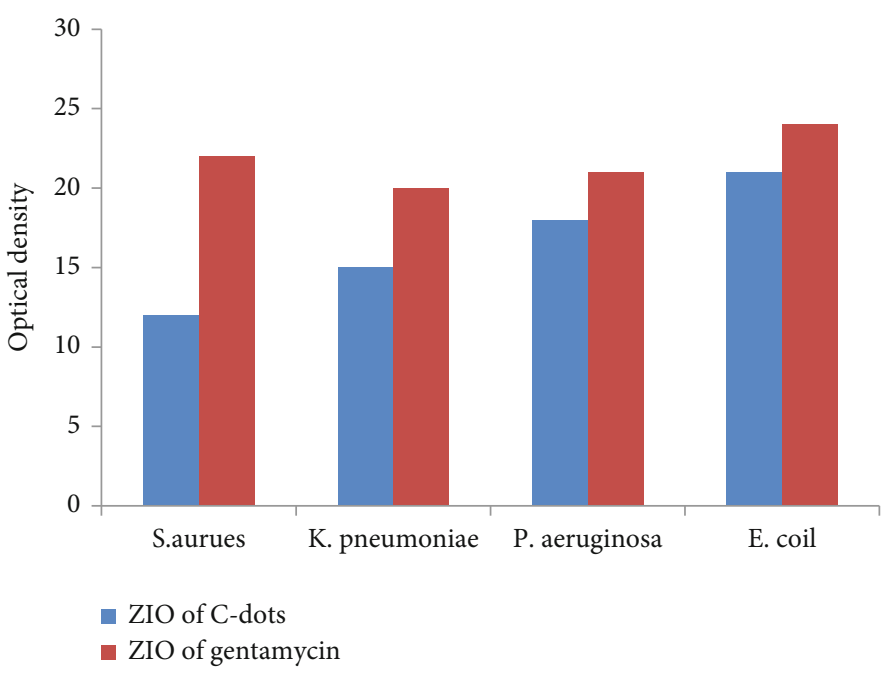

Figure 7: Zone of inhibition of microbial isolates.

TABLE 1: MIC test results of bacterial strains tested.

\begin{tabular}{lc}
\hline Microorganisms & MIC $(\mu \mathrm{g} / \mathrm{ml})$ \\
\hline S. aureus & 67 \\
K. pneumoniae & 72 \\
P. aeruginosa & 84 \\
E. coli & 89 \\
\hline
\end{tabular}

$\mathrm{C}=\mathrm{O} n-\pi^{*}$ transition. The result showed consistency with previous reports on the synthesis of C-dots $[18,19]$. Further, the emission characteristics of $\mathrm{C}$-dots obtained from the pomegranate peel were investigated with a photoluminescence spectrophotometer with excitation at $300 \mathrm{~nm}$, and Cdots exhibited a fluorescence emission band at $390 \mathrm{~nm}$ (Figure 3). These observations are in accordance with the results reported in literature [20]. The stability of the carbon dots at various $\mathrm{pH}$ was also investigated to understand the application potential of carbon dots, and it was observed to be stable in acidic $\mathrm{pH}$ and an emission band constantly observed at $390 \mathrm{~nm}$ for the carbon dots in the 2-7 pH range. However, the emission bands disappear or diminish with the increase in $\mathrm{pH}$ in the base medium. These could be due to protonation and deprotonation of hydronium ions from the surface of the carbon dots. These findings help in the detection of $\mathrm{pH}$ and as biomarkers in acidic medium.

FT-IR spectroscopy was used for the identification of functional groups present on the C-dots. C-dots obtained from the pomegranate peel showed absorption peeks at 3342.64 and $1650 \mathrm{~cm}^{-1}$ (Figure 4). These two peaks are due to the hydroxyl groups of the water molecule, and there were no any other major peaks observed in the spectra. This confirms that the solution contains only carbon dots and no other impurities are present in the solution. The particle size was obtained from DLS which showed a range of 5-9 nm (Figure 5). This confirms that C-dots were present in nanoform. The size distribution of carbon dots was seen to be from $1 \mathrm{~nm}$ to $50 \mathrm{~nm}$; however, the higher quantity of carbon dots was found to be between 5 and $9 \mathrm{~nm}$ in scale. These small-sized carbon dots are very much helpful in applications of carbon dots as biomarkers.

The transmission electron microscope was equipped to estimate and understand the size and shape of the carbon dots. It is observed that the carbon dots were found to be less than $10 \mathrm{~nm}$ in scale and shapes of the carbon dots were seen as spherical in nature (Figure 6). The size obtained through the TEM images supports the size confirmed through DLS. These results are in accordance with the production of carbon dots reported in literature $[14,21]$.

Microbial contamination of products from the farm to the table is a major source of concern in agriculture, as it costs producers money and puts the health of the final consumer at risk. Various investigations on the antibacterial effects of nanoparticles are currently being conducted [22].

The synthesized C-dots from pomegranate peels were investigated for their antimicrobial efficiency towards 4 pathogenic bacterial strains. The C-dot PP exhibits potent activity against selected isolates as shown in Figure 7, showing susceptibility towards $S$. aureus and K. pneumoniae, whereas it has moderate effect towards $P$. aeruginosa and resistance to E.coli when compared to ampicillin. MIC test results are represented in Table 1. The MIC was found to be high at $1000 \mu \mathrm{g} / \mathrm{ml}$. The extracts were efficient towards three isolates such as S. aureus (MIC-67 $\mu \mathrm{g} / \mathrm{ml}$ ), K. pneumoniae (MIC-72 $\mu \mathrm{g} / \mathrm{ml}$ ), and P. Aeruginosa (MIC-84 $\mu \mathrm{g} / \mathrm{ml}$ ). However, it did not show any efficacy towards E. coli. These results suggest that C-dots have efficacy as antimicrobial agents towards certain pathogens. The main processes implicated in C-dots' antibacterial effects are likely associated with the generation of reactive oxygen species, according to available data (ROS). Reactive oxygen species (ROS) are produced by C-dots and have been found to kill or inhibit bacteria [23]. The mechanism of action includes C-dot adhesion to the bacterial surface, photoinduced production of ROS, disruption and penetration of the bacterial cell wall/membrane, induction of oxidative stress with DNA/RNA damage, which leads to changes or inhibitions of important gene expressions, and induction of oxidative damage to proteins [24]. 


\section{Conclusion}

This study reported the effective utilisation of the agrowaste pomegranate peel as a prolific precursor for the production of carbon dots. The production of C-dots was investigated via a low-temperature method and was characterized with various analytical techniques. The synthesized C-dots were found to be around $5-9 \mathrm{~nm}$ in size which is of great advantage. The synthesized C-dots were investigated for their antimicrobial efficiency against pathogenic bacteria and found to have very strong activity. These results confirm that pomegranate peels are capable of producing C-dots for antimicrobial activity.

\section{Data Availability}

The data used to support the findings of this study are included within the article.

\section{Conflicts of Interest}

The authors declare no conflicts of interest.

\section{Acknowledgments}

This work was funded by King Saud University (Researchers Supporting Project number RSP-2021/27), Riyadh, Saudi Arabia.

\section{References}

[1] I. Singh, R. Arora, H. Dhiman, and R. Pahwa, "Carbon quantum dots: synthesis, characterization and biomedical applications," The Turkish Journal of Pharmaceutical Sciences, vol. 15, no. 2, pp. 219-230, 2018.

[2] M. Xue, W. Guan, W. Gu et al., "Microwave-assisted polyol synthesis of carbon nitride dots from folic acid for cell imaging," International Journal of Nanomedicine, vol. 9, pp. 5071-5078, 2014.

[3] A. Prasannan and T. Imae, "One-pot synthesis of fluorescent carbon dots from orange waste peels," Industrial \& Engineering Chemistry Research, vol. 52, no. 44, pp. 15673-15678, 2013.

[4] P. Y. Lin, C. W. Hsieh, M. L. Kung et al., "Eco-friendly synthesis of shrimp egg-derived carbon dots for fluorescent bioimaging," Journal of Biotechnology, vol. 189, pp. 114-119, 2014.

[5] H. Yi, Y. Huang, Z. C. Sha, X. H. Zhu, Q. Y. Xia, and H. Xia, "Facile synthesis of $\mathrm{Mo}_{2} \mathrm{~N}$ quantum dots embedded $\mathrm{N}$-doped carbon nanosheets composite as advanced anode materials for lithium-ion batteries," Materials Letters, vol. 276, article 128205, 2020.

[6] J. Singh, S. Kaur, J. Lee et al., "Highly fluorescent carbon dots derived from_Mangifera indica_leaves for selective detection of metal ions," Science of The Total Environment, vol. 720, article $137604,2020$.

[7] Y. Liang, L. X. Xu, K. Tang et al., "Nitrogen-doped carbon dots used as an "on-off-on" fluorescent sensor for $\mathrm{Fe}^{3+}$ and glutathione detection," Dyes and Pigments, vol. 178, article 108358, 2020.

[8] N. K. R. Bogireddy, J. Lara, L. R. Fragoso, and V. Agarwal, "One-step hydrothermal preparation of highly stable N doped oxidized carbon dots for toxic organic pollutants sensing and bioimaging," Chemical Engineering Journal, vol. 401, article 126097, 2020.
[9] C. H. Ding, Z. Q. Deng, J. C. Chen, and Y. Z. Jin, "One-step microwave synthesis of N,S co-doped carbon dots from 1,6hexanediamine dihydrochloride for cell imaging and ion detection," Colloids and Surfaces B: Biointerfaces, vol. 189, article 110838, 2020.

[10] G. Ferrentino, M. D. Asaduzzaman, and M. M. Scampicchio, "Current technologies and new insights for the recovery of high valuable compounds from fruits by-products," Critical Reviews in Food Science and Nutrition, vol. 58, no. 3, pp. 1-19, 2016.

[11] R. Das, R. Bandyopadhyay, and P. Pramanik, "Carbon quantum dots from natural resource: a review," Materials Today Chemistry, vol. 8, pp. 96-109, 2018.

[12] R. Atchudan, T. N. J. I. Edison, S. Perumal, N. Muthuchamy, and Y. R. Lee, "Hydrophilic nitrogen-doped carbon dots from biowaste using dwarf banana peel for environmental and biological applications," Fuel, vol. 275, article 117821, 2020.

[13] Z. X. Hu, X. Y. Jiao, and L. Xu, “The N,S co-doped carbon dots with excellent luminescent properties from green tea leaf residue and its sensing of gefitinib," Microchemical Journal, vol. 154, article 104588, 2020.

[14] J. Zhou, Z. Sheng, H. Han, M. Zou, and C. Li, "Facile synthesis of fluorescent carbon dots using watermelon peel as a carbon source," Materials Letters, vol. 66, no. 1, pp. 222-224, 2012.

[15] S. Kumar and K. Kannabiran, "Activite antifongique de Streptomyces VITSVK5 sp. sur des isolats d' Aspergillus mutir esistants isoles de patients atteints de tuberculose pulmonaire," Journal de Mycologie Médicale, vol. 20, no. 2, pp. 101-107, 2010.

[16] C. O. Okunji, C. N. Okeke, H. C. Gugnani, and M. M. Iwu, “An antifungal Spirostanol saponin from fruit pulp of Dracaena mannii," International Journal of Crude Drug Research, vol. 28, no. 3, pp. 193-199, 1990.

[17] H. Fan, M. Zhang, B. Bhandari, and C. H. Yang, "Food waste as a carbon source in carbon quantum dots technology and their applications in food safety detection," Trends in Food Science \& Technology, vol. 95, pp. 86-96, 2020.

[18] X. W. Tan, A. N. B. Romainor, S. F. Chin, and S. M. Ng, "Carbon dots production via pyrolysis of sago waste as potential probe for metal ions sensing," Journal of Analytical and Applied Pyrolysis, vol. 105, pp. 157-165, 2014.

[19] X.-Y. Jiao, L.-s. Li, S. Qin, Y. Zhang, K. Huang, and L. Xu, “The synthesis of fluorescent carbon dots from mango peel and their multiple applications," Colloids and Surfaces A: Physicochemical and Engineering Aspects, vol. 577, pp. 306-314, 2019.

[20] D. Kukreja, J. Mathew, R. Lakshmipathy, and N. C. Sarada, "Synthesis of fluorescent carbon dots from mango peels," International Journal of ChemTech Research, vol. 8, no. 5, pp. 61-64, 2015.

[21] R.-J. Fan, Q. Sun, L. Zhang, Y. Zhang, and A.-H. Lu, "Photoluminescent carbon dots directly derived from polyethylene glycol and their application for cellular imaging," Carbon, vol. 71, pp. 87-93, 2014.

[22] D. G. Panpatte and Y. K. Jhala, Eds., Nanotechnology for Agriculture: Crop Production \& Protection, Springer Nature, 2019.

[23] X. Dong, W. Liang, M. J. Meziani, Y. P. Sun, and L. Yang, "Carbon dots as potent antimicrobial agents," Theranostics, vol. 10, no. 2, pp. 671-686, 2020.

[24] M. M. Al Awak, P. Wang, S. Wang, Y. Tang, Y. P. Sun, and L. Yang, "Correlation of carbon dots' light-activated antimicrobial activities and fluorescence quantum yield," RSC Advances, vol. 7, no. 48, pp. 30177-30184, 2017. 